\title{
CBFB-MYH11 hypomethylation signature and PBX3 differential methylation revealed by targeted bisulfite sequencing in patients with acute myeloid leukemia
}

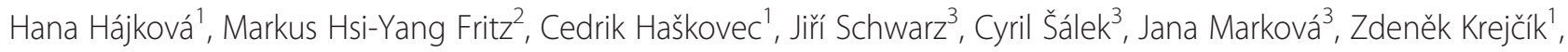
Michaela Dostálová Merkerová', Arnošt Kostečka ${ }^{1}$, Martin Vostrý ${ }^{1}$, Ota Fuchs ${ }^{1}$, Kyra Michalová ${ }^{4}$ Petr Cetkovský ${ }^{3}$ and Vladimír Beneš ${ }^{*}$

\begin{abstract}
Background: Studying DNA methylation changes in the context of structural rearrangements and point mutations as well as gene expression changes enables the identification of genes that are important for disease onset and progression in different subtypes of acute myeloid leukemia (AML) patients. The aim of this study was to identify differentially methylated genes with potential impact on AML pathogenesis based on the correlation of methylation and expression data.

Methods: The primary method of studying DNA methylation changes was targeted bisulfite sequencing capturing approximately 84 megabases (Mb) of the genome in 14 diagnostic AML patients and a healthy donors' CD34+ pool. Subsequently, selected DNA methylation changes were confirmed by 454 bisulfite pyrosequencing in a larger cohort of samples. Furthermore, we addressed gene expression by microarray profiling and correlated methylation of regions adjacent to transcription start sites with expression of corresponding genes.

Results: Here, we report a novel hypomethylation pattern, specific to CBFB-MYH11 fusion resulting from inv(16) rearrangement that is associated with genes previously described as upregulated in inv(16) AML. We assume that this hypomethylation and corresponding overexpresion occurs in the genes whose function is important in inv(16) leukemogenesis. Further, by comparing all targeted methylation and microarray expression data, PBX3 differential methylation was found to correlate with its gene expression. $P B X 3$ has been recently shown to be a key interaction partner of HOX genes during leukemogenesis and we revealed higher incidence of relapses in PBX3-overexpressing patients.
\end{abstract}

Conclusions: We discovered new genomic regions with aberrant DNA methylation that are associated with expression of genes involved in leukemogenesis. Our results demonstrate the potential of the targeted approach for DNA methylation studies to reveal new regulatory regions.

Keywords: Acute myeloid leukemia, CBFB-MYH11, DNA methylation, Targeted bisulfite sequencing, PBX3

\footnotetext{
*Correspondence: benes@embl.de

${ }^{2}$ European Molecular Biology Laboratory (EMBL), Core Facilities and Services,

Meyerhofstraße 1, Heidelberg, Germany

Full list of author information is available at the end of the article
} 


\section{Background}

Changes in DNA methylation patterns are a known hallmark of acute myeloid leukemia (AML) and underlie AML pathogenesis [1]. DNA methylation in patients with AML has been studied extensively and may reflect either specific molecular abnormalities or characterize a group of patients without an apparent molecular aberration. Specific translocations such as PML-RARA, AML1-ETO (RUNX1RUNX1T1), $M L L$ translocations or CBFB-MYH11 fusion, as well as CEBPA, NPM1, IDH1/IDH2, DNMT3A, TET2 and $R U N X 1$ mutations have been described to display distinct methylation signatures [2-4]. These epigenetic profiles are usually accompanied by specific gene expression features. Studying genes that are epigenetically deregulated in different groups of patients may contribute to a more detailed understanding of pathways involved in the leukemic transformation. Importantly, the effect of DNA methylation changes is greatly dependent on the location of differentially methylated regions (DMRs) [5]. New approaches using next-generation sequencing enable studying of DMRs scattered throughout the genome and targeted bisulfite sequencing offers a reasonably balanced ratio between cost and informativeness (number of CpGs covered) [6]. The link between gene expression and DNA methylation data is needed to find pathologically relevant DNA methylation changes, especially because many (or even the majority of) DMRs reflect the tissue of origin and not leukemia (cancer) specific changes [7].

In this study, 84 megabases $(\mathrm{Mb})$ of $14 \mathrm{AML}$ genomes and one CD34+ pool of cells from healthy donors were captured for DNA methylation and gene expression profiling. The aim was to identify differentially methylated genes with potential impact on AML pathogenesis based on the correlation of methylation and expression data.

AML patients with $C B F B-M Y H 11$ fusion $(C B F B$ - Corebinding factor, beta subunit; MYH11 - Myosin, heavy chain 11) resulting from inv(16) rearrangement clustered together in a hierarchal DNA methylation and expression analysis. The majority of differentially methylated regions unique for $C B F B-M Y H 11$ patients were hypomethylated and genes assigned to such regions were previously described as overexpressed in inv(16) AML [8].

$P B X 3$ (pre-B-cell leukemia homeobox 3), recently demonstrated as an important cofactor of HOXA9 in leukemogenesis [9], was validated as a gene whose gene expression levels correlated with DNA methylation of its putative regulatory region across AML subtypes. The importance of $P B X 3$ is underlined by the fact that $P B X 3$-overexpressing patients relapse more frequently. In summary, we discovered new genomic regions affected by aberrant DNA methylation that are associated with expression of genes implicated in leukemogenesis.

\section{Results}

Inv(16) methylation and expression cluster

We performed targeted bisulfite sequencing to discover specific methylation changes in 14 AML samples of diverse clinical and genetic background versus a pool of CD34+ healthy control cells (see Table 1 for patients' characteristics). Agilent's SureSelect ${ }^{\mathrm{XT}}$ Human MethylSeq system was used to interrogate DNA methylation of selected regions (84 megabases in total) of their genomes. These targeted regions comprise $\mathrm{CpG}$ islands, CpG shores and shelves, as well as cancer and tissuespecific DMRs. For all samples, on-target rates were very good (about 95\% for $+/-100 \mathrm{bp}$ ), while the percentage of targets above $20 \times$ coverage varied more widely (between $\sim 70 \%$ and $90 \%$ ). Targeted methylation data were correlated with previously obtained wholegenome bisulfite (WGBS) data (unpublished) for 3 cross-experiment (WGBS/target enrichment) samples. Taking into account positions with minimal coverage of 10 , there was a strong positive correlation $(R \geq 0.97)$ in all cases.

The hierarchal clustering analysis was done with the $\mathrm{R}$ package Pvclust [10] using the correlation as a distance measure and Ward's method. Only positions of autosomes (i.e. excluding mitochondria and sex chromosome calls) with coverage at least 10 and available for all samples $(n=2010310)$ were included in the analysis. From the clinical and molecular characteristics, only $C B F B$ MYH11 patients (samples 1 and 2) clustered together. In Figure 1A, clustering of all CpGs is shown (for separate clustering of CpGs inside or outside CpG islands see the Additional file 1: Figure S1A and S1B). None of the other molecular abnormalities formed clusters and we did not observe any effect of clinical status of AML (de novo, therapy-related, or AML with myelodysplasia-related changes). One of the inv(16) sample was derived from BM (sample 1) while the other (sample 2) from PB. Importantly, this had no effect on their clustering consistency. We investigated this uniformity between the results from $\mathrm{BM}$ and $\mathrm{PB}$ in more details using 454 pyrosequencing in a larger number of patients (see below).

Clustering of gene expression data for 8884 genes with detection $\mathrm{P}$ value $\leq 0.05$ in all 14 AML patients and 4 CD34+ healthy control cells confirmed the inv(16) cluster (Figure 1B). Consistent with the DNA methylation data, no other characteristics formed clusters. The selection of these 14 AML patients was done randomly throughout various clinical subgroups (de novo, secondary after MDS, secondary after breast cancer, AML with dysplasia) with the aim to find common DNA methylation changes among diverse AML subtypes. Therefore, we cannot make any general conclusions with regard to the existence/nonexistence of specific DNA methylation/gene expression profiles other than those demontrated in inv(16) AML, 
Table 1 Characteristics of AML patients

\begin{tabular}{|c|c|c|c|c|c|c|}
\hline Sample ID & Genetic aberration & Clinical characterization & FAB subtype & Gender & Material & $\%$ blasts \\
\hline AML_1 & CBFB-MYH11, FLT3-ITD & de novo AML & AML M4 & male & $\mathrm{BM}$ & 68 \\
\hline AML_2 & CBFB-MYH11 & de novo AML & AML M4 & female & PB & 40 \\
\hline AML_3 & NPM1 & therapy-related $\mathrm{AML}^{*}$ & AML M1 & female & PB & 67 \\
\hline AML_4 & MLL-PTD & therapy-related $\mathrm{AML}^{*}$ & AML M4 & female & BM & 42 \\
\hline AML_5 & NPM1, DNMT3A, FLT3-ITD & AML with multilineage dysplasia & AML M2 & female & PB & 58 \\
\hline AML_6 & none & AML with multilineage dysplasia & AML M1 & female & $\mathrm{BM}$ & 82 \\
\hline AML_7 & NPM1, DNMT3A, FLT3-ITD & de novo AML & AML M4 & male & $\mathrm{BM}$ & 94 \\
\hline AML_8 & FLT3-ITD & de novo AML & AML M1 & male & $\mathrm{BM}$ & 87 \\
\hline AML_9 & FLT3-ITD & de novo AML & AML M1 & male & $\mathrm{BM}$ & 89 \\
\hline AML_10 & NPM1, FLT3-ITD & de novo AML & AML M2 & female & $\mathrm{BM}$ & 75 \\
\hline AML_11 & MLL-PTD & de novo AML & AML M1 & male & $\mathrm{BM}$ & 92 \\
\hline AML_12 & MLL-PTD, CEBPA & de novo AML & AML M1 & female & PB & 80 \\
\hline AML_13 & NPM1, CEBPA & AML after MDS & AML M6 & male & PB & 60 \\
\hline AML_14 & none & AML after MDS & AML M2 & male & PB & 44 \\
\hline CD34+ & & pool of 4 CD34+ healthy control cells & & males & & \\
\hline
\end{tabular}

AML_1 to AML_14 are AML patients at diagnosis that were subjected to targeted bisulfite sequencing and gene expression profiling; CD34+ is a healthy control's pool; " after breast cancer; BM - bone marrow, PB - peripheral blood.

which were sufficiently verified in a validation cohort (see below).

\section{Hypomethylation signature of inv(16) AML patients}

We extracted genomic regions being uniquely differentially methylated in $C B F B-M Y H 11$ patients. There was a clear tendency towards hypomethylation with 125 out of 182 regions (69\%) displaying lower DNA methylation levels compared to the healthy donors' CD34+ pool of cells. All CBFB-MYH11 DMRs were uploaded to GREAT [11] and enrichment for genes previously described as upregulated in inv(16) AML patients was observed (reported in ref. [8], ID: VALK_AML_CLUSTER_9; Additional file 2: Table S1). This enrichment set comprised 10 genomic regions assigned to 6 genes - MN1, SPARC, ST18, DHRS3, FAM171A and BAHCC1 (see Additional file 3: Table S2). MN1, SPARC, ST18, FAM171A and DHRS3 were chosen for hypomethylation validation by 454 bisulfite pyrosequencing. As we were primarily interested in hypomethylation associated with overexpression, $B A H C C 1$ was excluded from the methylation/expression validation, because its expression levels were undetectable in AML as well as in healthy donors' samples according to microarray expression data. For MN1 and SPARC, two regions per gene were studied.

In summary, altogether 21 inv(16) AML, 15 non-inv(16) AML M4, 19 other AML (1 AML M0, 3 AML M1, 6 AML M2, 3 AML M3, 3 AML M5a, 2 AML M5b, 1 AML M6) and 10 healthy controls were examined. DNA methylation of individual regions (corresponding to individual amplicons) was expressed as an average DNA methylation of all CpGs in that particular region. Average levels of DNA methylation in assigned regulatory regions of MN1, SPARC, ST18 and DHRS3 were significantly lower for inv(16) versus non-inv(16) AML M4, other AML subtypes and healthy controls $(\mathrm{P}<0.0001)$ (see Figure 2). Sequencing of $F A M 171 A$ failed twice in all samples probably due to the low complexity of the amplicon (because of the long stretches of identical bases - homopolymers) introduced after bisulfite conversion and FAM171A was therefore excluded from further analysis. For $M N 1$, both of the studied regions displayed lower levels of methylation (for MN1_region_2 hypomethylation graph see Additional file 4: Figure S2A). For SPARC, the second studied region (SPARC_region_2) had low DNA methylation levels in $\operatorname{inv}(16)$ as well as in other AML and healthy donors (see Additional file 4: Figure S2B). Therefore only methylation of region 1 has a potential impact on SPARC expression. The 454-pyrosequencing results point to the site-specific CBFB-MYH11 hypomethylation signature of genomic regions assigned to MN1, SPARC, ST18 and DHRS3.

As we used either $\mathrm{PB}$ or $\mathrm{BM}$ as a starting material, we investigated whether there is a concordance between DNA methylation results from $\mathrm{PB}$ and $\mathrm{BM}$ in $10 \mathrm{AML}$ patients with both materials at diagnosis available. We found high correlation between $\mathrm{PB}$ and $\mathrm{BM}$ results for all of the studied regions $(R=0.96)$.

Further, we evaluated expression levels of MN1, SPARC, ST18 and DHRS3 by TaqMan gene expression assays in all samples already examined by 454 pyrosequencing. As can be seen in Figure 3, inv(16) patients had higher average 

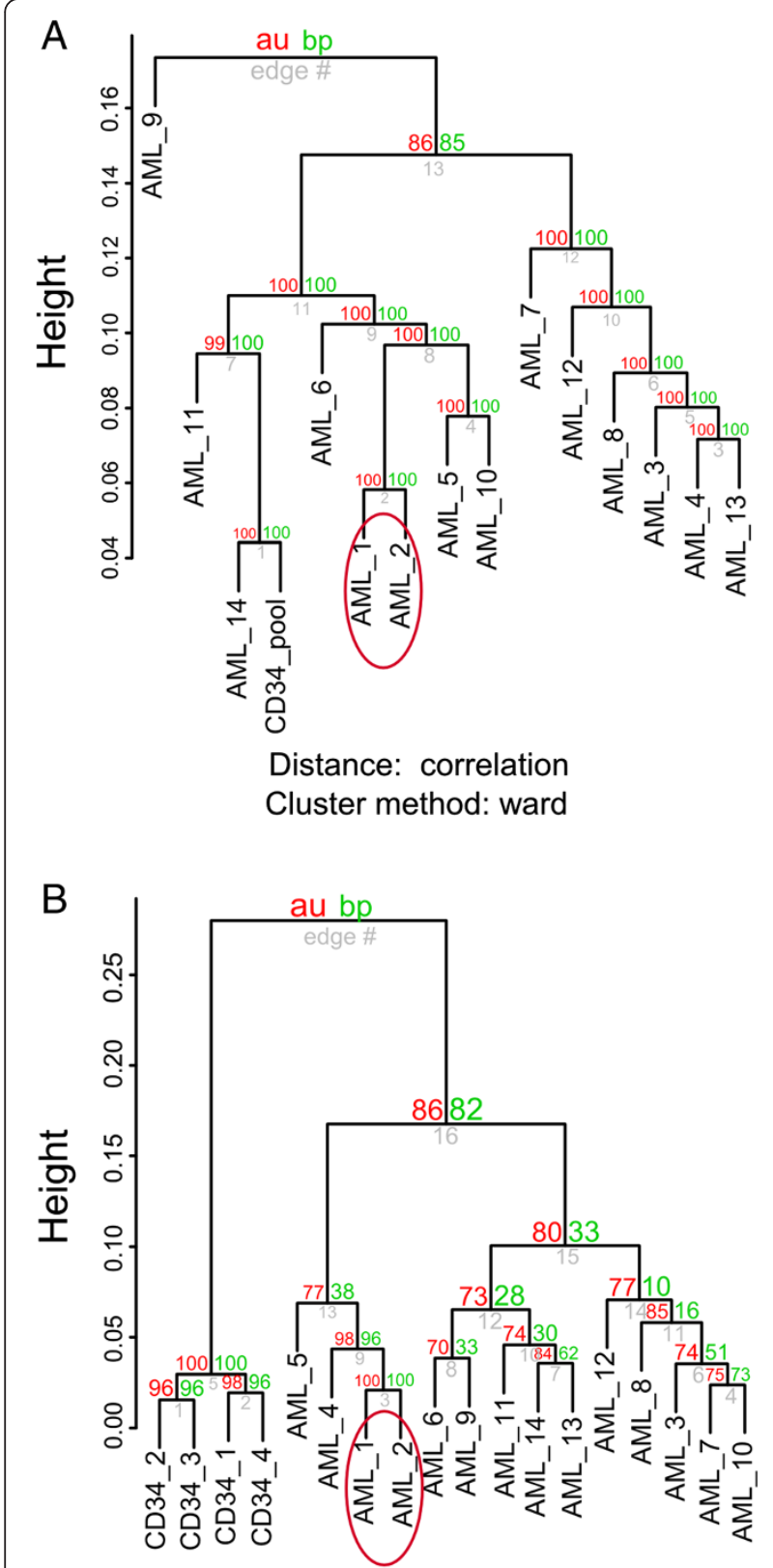

Distance: correlation Cluster method: ward

Figure $1 \operatorname{lnv}(16)$ methylation and expression cluster. (A) Hierarchal DNA methylation clustering of CpGs ( $n=2010$ 310) using the correlation as a distance measure and Ward's method (AML_1 to AML_14 - AML patients; CD34_pool - healthy control's CD34+ pool) indicating CBFB-MYH11 methylation cluster (in ellipse), other molecular abnormalities did not form clusters neither clinical characteristics did it; (B) Hierarchal gene expression clustering of altogether 8884 genes with a detection $P$ value $\leq 0.05$ in all $14 \mathrm{AML}$ patients (AML_1 to AML_14) and 4 CD34+ healthy control cells confirmed inv(16) cluster (in ellipse). levels of expression for all 4 genes in comparison to other non-inv(16) AML samples, but only in ST18 when compared to healthy donors.

Accordingly, ST18 was the most overexpressed gene in inv(16) AML compared to CD34+ cells of healthy controls in the microarray expression data. However, expression for all 3 remaining genes - MN1, SPARC, and DHRS3 was also upregulated in inv(16) AML (approximately 3-times higher than in CD34+ cells of healthy controls). Due to this inconsistency, we decided to re-measure gene expression by the use of SybrGreen RQ-PCR. This approach gave us results more similar to those observed by Illumina expression microarrays. As seen in Figure 4, differences in the gene expression levels between inv(16) AML and healthy controls increased significantly for MN1 and SPARC. For DHRS3, the ratio of expression between inv (16) and healthy donors changed only slightly, and for ST18 remained the same. One possible explanation of this discrepancy is the genomic location of primers. SybrGreen RQ-PCR primers for MN1 and SPARC are located within the same exons as are the probes on the Illumina expression microarray, while TaqMan primers for MN1 and SPARC have different, exon-exon locations. On the contrary, both types of primers (TaqMan and SybrGreen) for ST18 and DHRS3 have exon-exon locations. We checked SybrGreen RQ-PCR products specificity by melting analysis in all samples and no unspecificity was detected. It seems that in this particular case the selection of PCR detection system may influence results in a considerable manner. In general, this issue deserves further exploration.

In summary, ST18 is the only overexpressed gene in inv(16) compared to other AML subtypes and healthy donors, irrespectively of the RQ-PCR system used.

\section{Correlation of methylation data with expression}

For each transcription start site (TSS), a window was defined from $5 \mathrm{~kb}$ up- to $1 \mathrm{~kb}$ downstream of that TSS. Targeted regions overlapping this window were associated with the gene of the given TSS. GENCODE $v 14$ gene annotation was used for assigning DNA methylation of target regions to corresponding genes. Expression and methylation data were then correlated using Spearman rank tests. The small sample size did not allow for filtering based on $\mathrm{P}$ values and thus an ad hoc measure was employed, requiring strong anti-correlation $(\mathrm{R} \leq-0.7)$, and a change of methylation between control and at least one AML sample 2-fold or greater (with either the control or at least one of the AMLs having methylation ratio 0.3 or greater). These strict filtering parameters resulted in a list of 163 genomic regions assigned to 130 unique genes (see Additional file 5: Table S3). Among these genes, we 

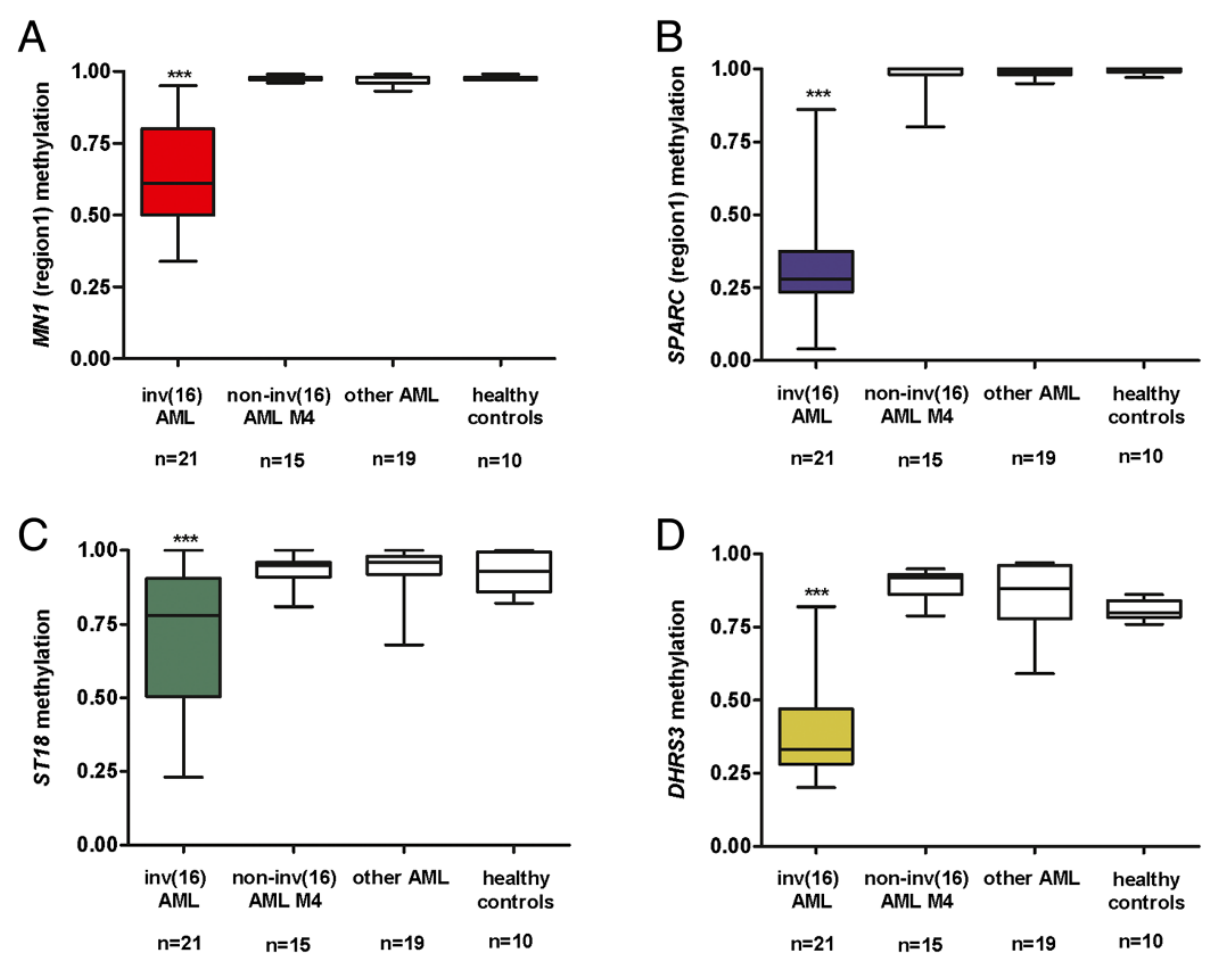

Figure 2 Hypomethylation signature in inv(16) AML. Hypomethylation of MN1 (A), SPARC (B), ST18 (C) and DHRS3 (D) regulatory regions in inv(16) patients compared to AML M4 without inv(16), other AML subtypes and healthy controls; asterisks correspond to statistically significant changes of expression in inv(16) patients versus other groups, ${ }^{* * *} \mathrm{P}<0.0001$.

chose $P B X 3$ and GFI1 to validate the association between DNA methylation and gene expression changes observed in this cohort of 14 AML patients.

\section{PBX3 and GFI1 differential methylation is involved in their} expression regulation

The DMRs associated with $P B X 3$ are located downstream ( -160 to $-1451 \mathrm{bp}$ ) of annotated CpG island (for exact location see Additional file 5: Table S3). We focused on a DMR encompassing a TAF1 binding site (chr9:128 510 974-128 511259 according to GRCh37/hg19). TAF1 encodes the largest subunit of TFIID and this subunit binds to core promoter sequences encompassing TSSs.

We measured the expression of PBX3 mRNA in 123 AML diagnostic samples and 15 healthy controls (for a graph see Additional file 6: Figure S3). 24\% of AML had down- and 22\% upregulated PBX3 expression (with a minimum change in the expression of more than 2-fold and at the same time of more than one order of magnitude from healthy donors' $P B X 3$ average expression). 454 pyrosequencing established the role of DNA methylation in down- and upregulation of $P B X 3$ in $30 \mathrm{AML}$ patients at diagnosis (all of them with blast count $\geq 60 \%$; Figure 5). Elevated levels of $P B X 3$ were connected with hypomethylation of a regulatory region (median methylation level 0.25 , range $0.15-0.36 ; \mathrm{P}<0.0001$ ), whereas decreased levels of expression with hypermethylation (median methylation level 0.51, range $0.31-0.98 ; \mathrm{P}=$ 0.002). Control samples of healthy donors and AML samples with normal $P B X 3$ expression displayed intermediate levels of methylation (median 0.35, range 0.19 0.51). These results demonstrate the link between $P B X 3$ expression and DNA methylation levels of the $P B X 3$ regulatory region located downstream of the $P B X 3$ annotated CpG island.

Regarding GFI1, we examined a 409 bp long region within the GFI1 promoter (chr1:92 952 229-92 952 637). Average DNA methylation levels of healthy controls $(\mathrm{n}=14)$ fluctuated around $3.86 \%$. Very similar to that, DNA methylation levels of AML within normal gene expression range were $4.54 \%$. Only a minority of AML samples (3/64) showed decreased GFI1 expression levels. However, all 3 GFI1-downregulated patients displayed higher methylation levels - 22.8\%, 28.9\% and $19 \%$, respectively. On the contrary, upregulation of GFI1 was not connected with any significant changes of DNA methylation levels, probably due to the fact that normal DNA methylation levels were already low, therefore any further decrease would not have a functional role. 

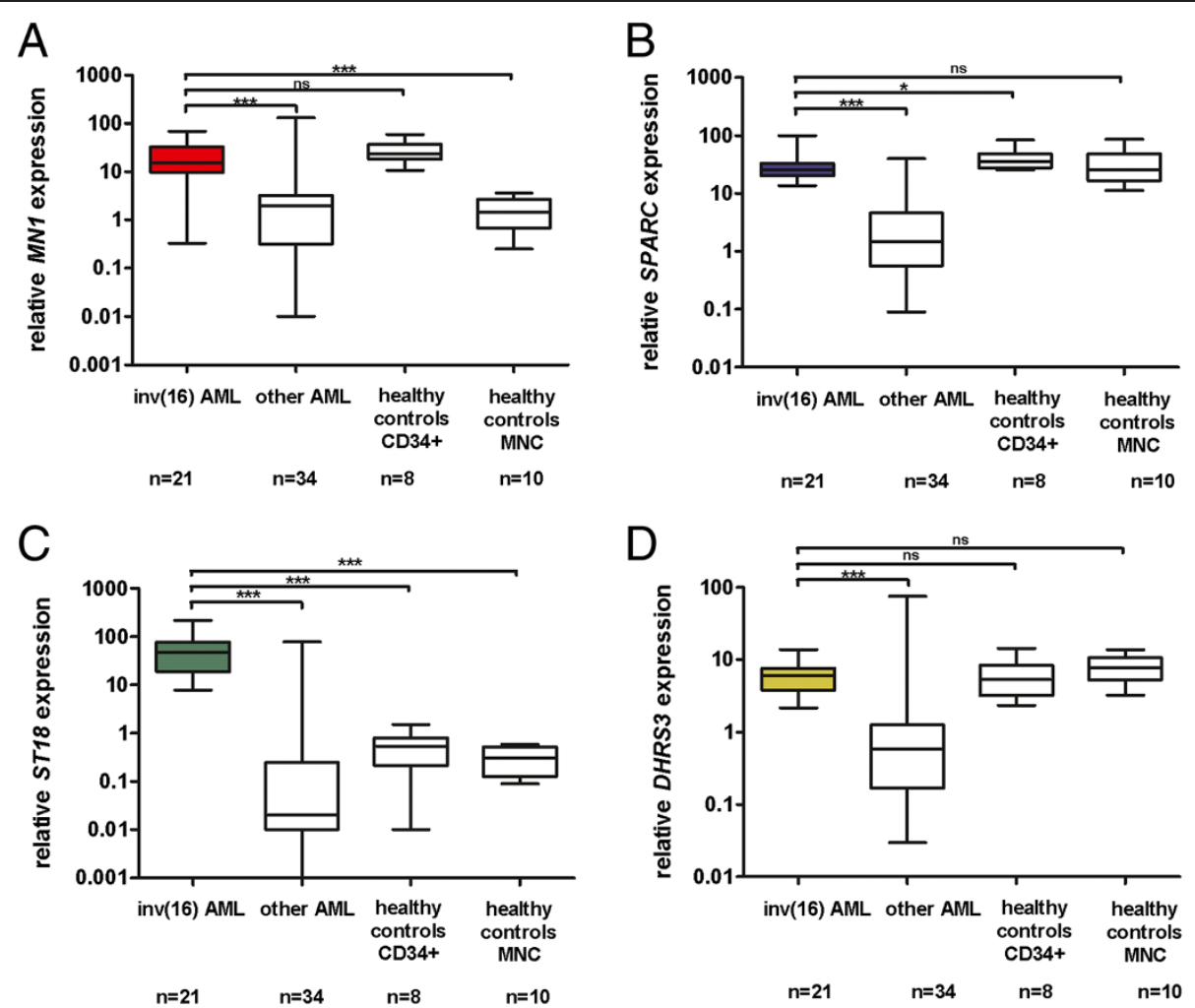

Figure 3 Expression of hypomethylated genes in inv(16) AML measured by TaqMan gene expression assays. Relative expression value of MN1 (A), SPARC (B), ST18 (C) and DHRS3 (D) in inv(16) AML patients versus other AML subtypes and healthy control cells; MNC - mononuclear cells; asterisks correspond to statistically significant changes of expression, ${ }^{* *} \mathrm{P}<0.0001,{ }^{*} \mathrm{P}<0.05$, ns - not significant.

\section{PBX3 expression levels and their impact on prognosis of AML patients}

Because $P B X 3$ is one of the four genes, whose common expression signature was described as having an impact on overall survival (OS) in AML patients [12], we decided to evaluate its expression levels in terms of OS and relapse-free survival (RFS). Only patients receiving standard curative therapy and those who did not die during the first induction were included in this analysis. Altogether 40 AML patients were assessed, 21/40 had low and 19/40 had high expression levels. Low and high expression levels were defined as a change in expression of at least one order of magnitude as well as 2-fold from healthy donors' average $P B X 3$ expression.

We did not observe any effect of $P B X 3$ expression levels on $\mathrm{OS}$ in AML patients (Figure 6A), however we found higher relapse rates in AML patients with overexpressed $P B X 3$ (Figure 6B, $\mathrm{P}=0.004$ ).

We also performed multivariate analysis for both OS and RFS. Firstly, we tested the following parameters by univariate analysis: age, white blood count (WBC), complete remission after induction therapy (CR after induction), good/intermediate/poor prognosis according to cytogenetics, $P B X 3$ expression and FLT3-ITD status. For OS, only CR after induction was statistically significant in both univariate and multivariate analyses $(\mathrm{P}<0.001)$. For RFS, parameters significant in univariate testing (WBC, CR after induction, FLT3-ITD status and $P B X 3$ expression) were evaluated by Cox regression. Only $C R$ after induction and $P B X 3$ expression retained statistical significance $(\mathrm{P}=0.002$ and $\mathrm{P}=0.028$, respectively) in multivariate testing.

Furthermore, we evaluated whether there is an association between $P B X 3$ expression levels and presence of $M L L$-PTD or belonging to prognostically adverse AML subgroup. No such correlation was found, on the other hand there was no $P B X 3$-overexpressing patient among AML patients within the prognostically favourable subgroup (0/6 cases from prognostically favourable versus $26 / 46$ cases from prognostically intermediate or adverse subgroup overexpress $P B X 3,0 \%$ versus $57 \%$ respectively, $\mathrm{P}=0.02$ ). Leukemic transformation mediated by MLLfusion proteins has been suggested to be dependent on the presence of $P B X 3$ expression [9]. In our cohort, all three $M L L$-translocated patients, two with $M L L T 3-M L L$ (MLL-AF9) and one with MLL-MLLT1 (MLL-ENL), had upregulated $P B X 3$ expression, but the low number of $M L L$ translocations limited statistical testing of this association. 

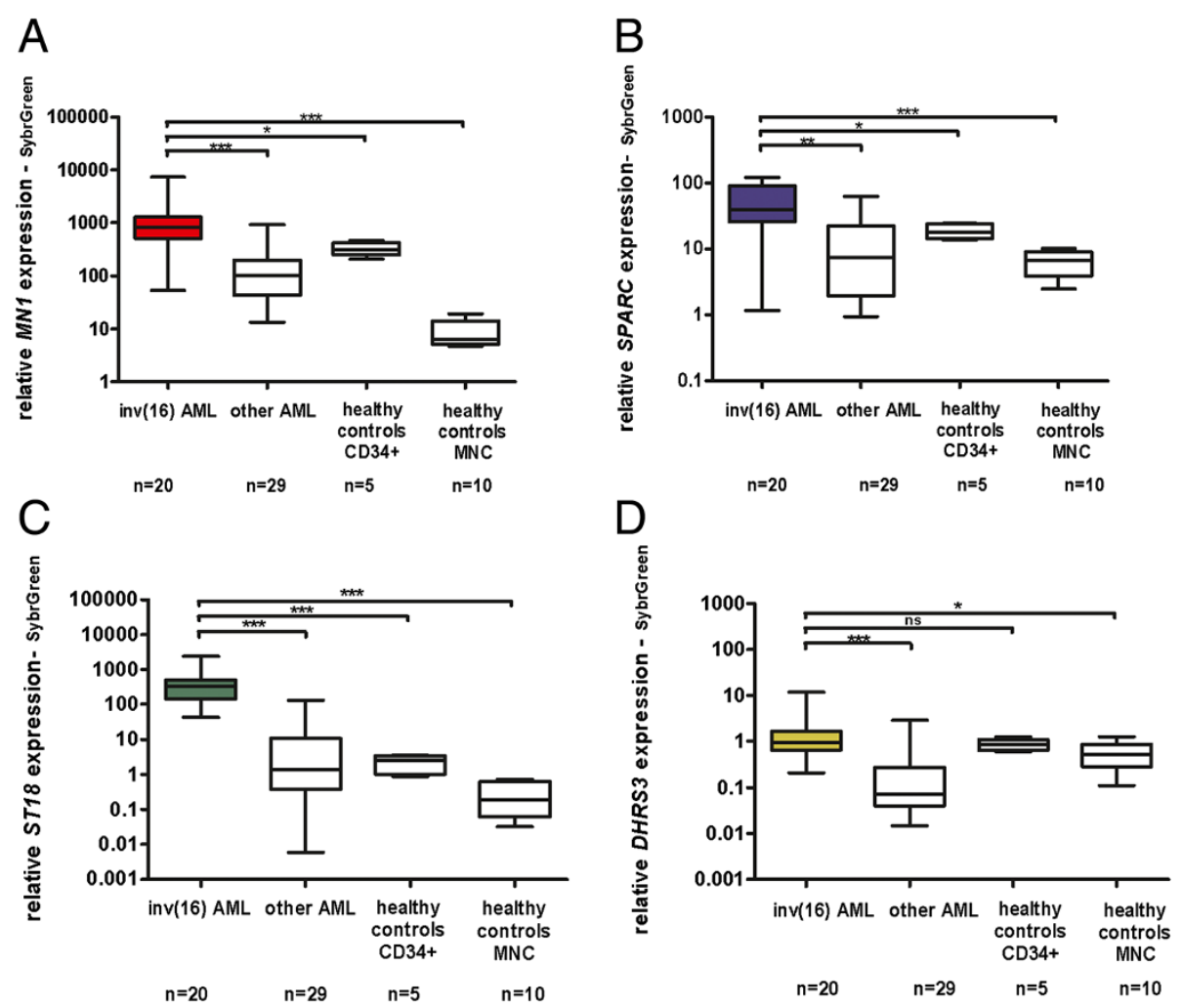

Figure 4 Expression of hypomethylated genes in inv(16) AML measured by SybrGreen RQ-PCR. Relative expression value of MN1 (A), SPARC (B), ST18 (C) and DHRS3 (D) in inv(16) AML patients versus other AML subtypes and healthy control cells; MNC - mononuclear cells; asterisks correspond to statistically significant changes of expression, ${ }^{* *} \mathrm{P}<0.0001,{ }^{*} \mathrm{P}<0.05,{ }^{* * P}<0.01$, ns - not significant.

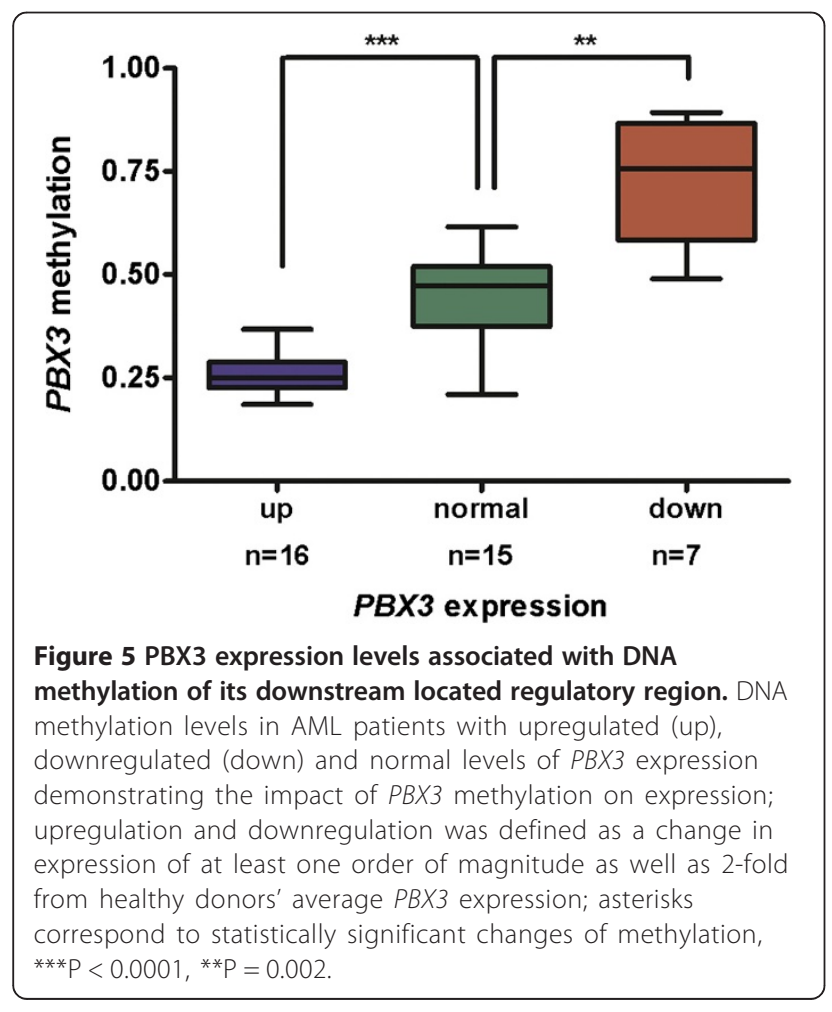

\section{Discussion}

Here, we report a $C B F B-M Y H 11$, i.e. inv(16), specific hypomethylation that may play a role in upregulation of some previously described inv(16) overexpressed genes [8]. We analysed DNA methylation and expression data of MN1, SPARC, ST18 and DHRS3 in 55 AML patients and 10 healthy controls. Lower methylation levels of these genes in inv(16) patients versus other AML and healthy donors were confirmed. When measured by TaqMan gene expression assays, inv(16)-specific overexpression of $M N 1$, SPARC, ST18 and DHRS3 was found with respect to noninv(16) AML, but only in ST18 in comparison with healthy donors. This was inconsistent with the Illumina microarray expression data as well as previously published data [8]. Therefore, we re-measured the results using SybrGreen RQ-PCR and we obtained different values. In this RQPCR experiment, changes in expression levels (between inv(16) AML and healthy donors) were also significant for MN1 and SPARC. We excluded both the role of PCR nonspecificity and DNA contamination. Interestingly, $M N 1$ and SPARC primers for SybrGreen RQ-PCR, and MN1 and $S P A R C$ probes on the expression microarray, are localized within the same exons, while MN1 and SPARC TaqMan probes have different, exon-exon localizations. We cannot claim that this is the only reason for the 

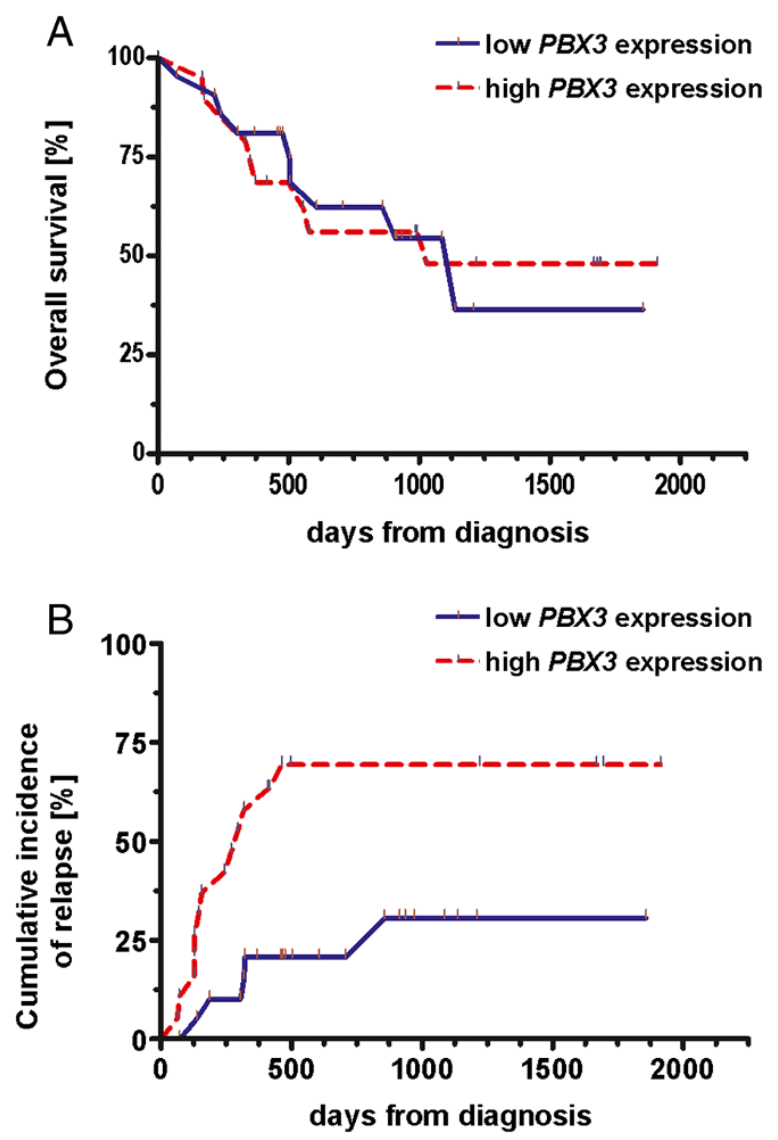

Figure 6 Impact of $P B X 3$ expression levels on prognosis. (A) Kaplan-Meier curves of overall survival (OS) in AML with low versus high expression of $P B X 3$ displaying no significant difference between these two groups of AML patients; (B) Cumulative incidence of relapse showing higher relapse rates in $P B X 3$-overexpressing $A M L$ patients $(P=0.004)$

different results, this issue definitively needs deeper examination in future studies. It is of interest that the results obtained by SybrGreen RQ-PCR are in agreement with the publicly available data (GSE34823) of the study of Bletiere et al. [13].

Average gene expression levels of MN1, SPARC and ST18 extracted from the above-mentioned dataset are higher in inv(16) AML in comparison with healthy donors' bone marrow (4-times for MN1 and SPARC, 7-times for ST18), and DHRS3 expression is basically the same in both groups.

Further, we extracted the data from The Cancer Genome Atlas (TCGA, https://tcga-data.nci.nih.gov/tcga/) and they also confirmed a link between hypomethylation of MN1 and DHRS3 regulatory regions and their overexpression in inv(16) AML when compared with AML samples with normal karyotype (healthy controls data were not available). Regulatory regions corresponding to remaining genes lacked information of their methylation status in
TCGA due to the absence of appropriate CpGs in HumanMethylation450 BeadChip used at TCGA study [3]. This supports the profitability of studying DNA methylation using targeted bisulfite sequencing, which provides more complex coverage than microarray based techniques.

The hypomethylation pattern that we discovered in inv(16) AML patients is remarkable also with respect to the very recently published data of Mandoli and colleagues [14]. For the first time, their study revealed the involvement of CBFB-MYH11 not only in repression but as well in transcriptional activation. The direct involvement of CBFB-MYH11 in overexpression of MN1, ST18 and SPARC is supported with 2-fold downregulation of these genes upon $C B F B-M Y H 11$ knockdown as reported in their work. However, none of the 1874 high-confidence CBFB-MYH11 binding sites [14] overlaps with any of the hypomethylated regions reported here. DHRS3 was among the genes upregulated upon CBFB-MYH11 knockdown, which is in agreement with its disputable upregulation in inv(16) AML. There were great differences in localization of hypomethylated regions with respect to TSSs of individual genes. With regard to $M N 1$ and SPARC, the hypomethylation was located not far from their TSSs (for location see Additional file 3: Table S2), which makes the assumption of their role in the expression of these genes more straightforward. Moreover, MN1 and SPARC potential regulatory regions overlap regions of active chromatin (enhancers) in mobilized CD34+ cells as observed in the EpiGenome Browser (http://epigenomegateway.wustl.edu/ browser) suggesting a role of these regions in transcription regulation. On the contrary, differentially hypomethylated sites assigned by GREAT to ST18 and DHRS3 are placed much farther from their TSSs, specifically approximately 275 kilobases $(\mathrm{kb})$ downstream for ST18 and $277 \mathrm{~kb}$ upstream for DHRS3. ST18 and DHRS3 assigned regulatory regions are placed within chromatin marked with low transcription activity and enhancer, respectively (in mobilized CD34+ cells, data from EpiGenome Browser).

MN1 expression levels have been shown to stratify prognosis of cytogenetically normal (CN) AML patients and its overexpression is connected with a poor outcome of CN-AML patients $[15,16]$. Nevertheless, inv(16) AML patients are generally associated with a good prognosis $[17,18]$ in spite of their frequent MN1 overexpression. Functional studies have proved that overexpression of MN1 cooperates with inv(16) in developing AML in vivo and that neither inv(16) or MN1 alone are capable of promoting leukemia [19]. According to our results it seems that hypomethylation is present uniquely in inv (16) AML patients in both MN1 assigned regions (none of the other AML subtypes or healthy controls displayed MN1 hypomethylation in our cohort). So it supports the theory that upregulation of some genes that might involve 
MN1 is crucial for inv(16) leukemogenesis and hypomethylation may be therefore needed to ensure stable overexpression of critical genes. Apparently the mechanism of MN1 upregulation is different in non-inv(16) AML patients or potentially hypomethylation of other regulatory areas located elsewhere may be involved.

Correlation between targeted DNA methylation and microarray expression data of 14 AML patients and a healthy controls' CD34+ pool revealed $P B X 3$ differential methylation and gene expression. $P B X 3$ (pre-B-cell leukemia homeobox 3) is part of the three amino acid loop extension (TALE) family of transcription factors, which include the products of the Pbx and Meis genes and are capable of heterodimerization with the Hox proteins [20]. Recently, $P B X 3$ was reported to have a synergistic effect with $H O X A 9$ in transforming normal hematopoietic progenitor cells in vitro as well as in vivo [8]. Moreover, $P B X 3$ is one of the four genes (HOXA6, HOXA9, PBX3 and MEIS1), whose common expression signature was shown to influence overall survival in CN-AML [12]. All evidence points to an important role of $P B X 3$ in leukemogenesis. This is the first report uncovering DNA methylation as a plausible regulator of $P B X 3$ expression. We found a strong negative correlation between levels of $P B X 3$ methylation and expression in 8 healthy donors' samples and 30 AML patients at diagnosis $(\mathrm{P}<0.0001$ and $\mathrm{P}=0.002$ for upregulation and downregulation, respectively). Localization of $P B X 3$ differential methylation overlaps TAF1 binding site according to ENCODE ChIP-Seq data from UCSC genome browser. TAFs (TBP-associated factors) create a stable complex with TBP (TATA-binding protein) and RNAPII to form a preinitiation complex, so we may assume that DNA methylation status of TAF1 binding site can directly influence the accessibility of DNA for transcription enzymes. The probability of transcription initiation is possibly dependent on whether the DNA methylation is low with high expression rates or DNA methylation is high with decreased expression or finally intermediate DNA methylation corresponding to in-between expression levels. As $P B X 3$ has a CpG island (CGI) overlapping its TSS, we also looked at its methylation status. Based on targeted methylation data, there was no methylation present either in AML or healthy controls. Therefore, methylation status of downstream located control element rather than CGI methylation is most likely crucial for $P B X 3$ expression. Further we focused on potential prognostic significance of $P B X 3$ expression in terms of overall survival (OS) and incidence of relapse. High $P B X 3$ expression levels were not related to different $\mathrm{OS}$ compared to $\mathrm{AML}$ patients with low $P B X 3$ expression, however relapse rates were significantly higher in $P B X 3$-overexpressing patients by both univariate and multivariate testing. This suggests more aggressive phenotype/course of disease of these patients, which is not reflected in the OS probably due to the early and effective treatment of relapses - often followed by bone marrow transplantation. We also showed that $P B X 3$ overexpression did not occur in AML patients within cytogenetically favourable subgroup.

We validated the methylation/expression correlation stated in the Additional file 5: Table S3 also for GFI1. Moreover, the observed correlations are further supported by the presence of genes, for which the role of DNA methylation is already published such as $M P O$ [21], CEBP $\alpha, D A P K 1, I R F 8$ and PRDX8 [22,23].

\section{Findings}

We found a new hypomethylation signature specific for inv(16) AML patients that may be responsible for overexpression of some genes that are crucial for inv(16) pathogenesis. MN1 gene is likely to be a key gene involved in the pathogenesis of inv(16) AML and hypomethylation in the regulatory region near its TSS in inv(16) AML patients was confirmed, even on the basis of publicly available data from TCGA. Furthermore, we explored new regulatory region for $P B X 3$ and association of its methylation with $P B X 3$ expression. Therefore, targeted bisulfite sequencing represents a convenient approach in terms of genome coverage and informativeness with a great potential to reveal new regulatory regions of genes involved in leukemic transformation.

\section{Materials and methods \\ Patients}

For targeted bisulfite sequencing, 14 AML patients at diagnosis (see Table 1) and pooled CD34+ cells from 4 healthy donors were sequenced. Genes selected based on targeted bisulfite sequencing results were examined using 454 bisulfite pyrosequencing in a larger cohort of AML patients (their characteristics are given in Additional file 7: Table S4). Informed consent was obtained from all patients and healthy blood donors enrolled in the study. The study was approved by the IHBT Institutional Ethics Committee according to the Helsinki Declaration.

\section{Sample preparation}

Mononuclear cells (MNC) from peripheral blood (PB) or bone marrow $(\mathrm{BM})$ of the AML patients at diagnosis were separated by Ficoll gradient centrifugation (Histopaque, Sigma-Aldrich, Steinheim, Germany). CD34+ cells were harvested from buffy coats of healthy blood donors using MicroBead kits (Miltenyi Biotec $\mathrm{GmbH}$, Bergish Gladbach, Germany). The CD34+ pool was created by mixing of 4 individual healthy blood donors' separated cells (all of them men aged 42 to 58 years old, median age 45.5). DNA and RNA were extracted using AllPrep DNA/RNA Mini Kit (Qiagen, Hilden, Germany). Bisulfite 
conversion was performed from $1 \mu \mathrm{g}$ of DNA by EpiTect Bisulfite Kit (Qiagen) and eluted into $40 \mu \mathrm{l}$ of EB buffer. cDNA was prepared using M-MLV RT (Moloney Murine Leukemia Virus Reverse Transcriptase, Promega, Madison, WI, USA).

\section{Targeted bisulfite sequencing}

Preparation of targeted bisulfite libraries started with $3 \mu \mathrm{g}$ of genomic DNA and was carried out using SureSelect ${ }^{\mathrm{XT}}$ Human Methyl-Seq kit (Agilent, Agilent Technologies, Santa Clara, CA, USA) according to the manufacturer's instructions. Libraries were multiplexed into 4 pools and each pool was sequenced on $2 \mathrm{HiSeq}^{\mathrm{Tm}} 2000$ (Illumina, San Diego, CA, USA) lanes using 105 bp paired-end sequencing reads with average coverage of 83 - ranging from 46 to 131 .

\section{4 bisulfite pyrosequencing}

Bisulfite-treated (BS) DNA was subjected to 2-round PCR. In the 1st round of PCR, loci-specific primers with M13 universal tails were used to amplify regions of interest. Subsequently, primers specific to M13 universal tails now tailed with 454 -specific sequencing primers and a unique barcode sequence (MID) were applied to the 2nd PCR. Loci-specific primers were designed with Methyl Primer Express v1.0 software (Applied Biosystems Inc. Foster City, CA, USA; see Additional file 8: Table S5 for primer sequences). HotStarTaq DNA polymerase (Qiagen) and manufacturer's recommended PCR reaction conditions were used for amplification. $2 \mu \mathrm{l}$ of BS DNA was added to the 1 st PCR and $1 \mu \mathrm{l}$ of $100 \times$ diluted 1st round PCR product was subjected to the 2 nd PCR. PCR cycling conditions were as follows: 1st round PCR - initial denaturation $\left(15 \mathrm{~min}\right.$ at $\left.95^{\circ} \mathrm{C}\right)$, followed by 35 cycles of denaturation $\left(30 \mathrm{~s}\right.$ at $\left.94^{\circ} \mathrm{C}\right)$, annealing (30s at $\mathrm{Ta}{ }^{\circ} \mathrm{C}, \mathrm{Ta}$ - annealing temperature, see Additional file 8: Table S5) and extension ( $1 \mathrm{~min}$ at $\left.72^{\circ} \mathrm{C}\right)$, final extension $\left(10 \mathrm{~min}\right.$ at $\left.72^{\circ} \mathrm{C}\right)$; 2 nd round of PCR - initial denaturation $\left(15 \mathrm{~min}\right.$ at $\left.95^{\circ} \mathrm{C}\right)$, followed by 26 cycles of denaturation $\left(30 \mathrm{~s}\right.$ at $\left.94^{\circ} \mathrm{C}\right)$, annealing $\left(30 \mathrm{~s}\right.$ at $\left.60^{\circ} \mathrm{C}\right)$ and extension $\left(1 \mathrm{~min}\right.$ at $\left.72^{\circ} \mathrm{C}\right)$, final extension $\left(5 \mathrm{~min}\right.$ at $\left.72^{\circ} \mathrm{C}\right)$. All amplicons after 2 nd round of PCR (up to 288 for one run) were purified using Agencourt AMPure ${ }^{\mathrm{XP}}$ magnetic beads (Beckman Coulter, Fullerton, CA, USA) and Biomek ${ }^{\odot}$ FXP Laboratory Automation Workstation (Beckman Coulter). Precise concentration of amplicons were determined using Quant-iT ${ }^{\mathrm{Tm}}$ PicoGreen dsDNA Assay Kit (Life Technologies, Carlsbad, CA, USA) and amplicons were equimolarly pooled to obtain amplicon library with $10^{9}$ fragments/ $\mu$ l concentration. Subsequent procedures were carried out according to 454 amplicon sequencing manuals (454 Life Sciences, Roche Applied Science, Branford, CT, USA) on the GS Junior sequencer (Roche). An average overall coverage of 225 reads was observed (ranging from 53 to 659 for individual amplicons).

\section{mRNA microarray profiling}

Gene expression profiles from 14 AML patients and 4 CD34+ cells of healthy controls were generated by HumanHT-12 v4 Expression BeadChip Kit (Illumina). The chip scanning was done with a BeadStation 500 instrument (Illumina).

\section{Quantitative real-time PCR (RQ-PCR)}

The expression levels of selected genes were assessed with TaqMan Gene Expression Assays (Life Technologies) - see Additional file 9: Table S6 for individual Assay IDs. GAPDH was utilized as a house-keeping gene. Amplification was carried out with TaqMan Universal Master Mix II (Life Technologies) and recommended cycling conditions. SybrGreen RQ-PCR was performed using QuantiTect SYBR Green PCR Kit (Qiagen) and pre-designed $\mathrm{KiCqStart}^{\oplus} \mathrm{SYBR}^{\circledR}$ Green primers (Sigma-Aldrich). Each sample was run in duplicates on a StepOne instrument (Life Technologies).

\section{Molecular genetics}

The presence of internal tandem duplication (ITD) in the juxtamembrane (JM) and tyrosine kinase 1 (TKD1) domains (exons 12-14) of FLT3 gene and the presence of $C B F B-M Y H 11$ fusion transcript at diagnosis was detected as described previously [24]. Further, we examined mutations in NPM1 [25], CEBPA [26] and DNMT3A [27] and intragenic $M L L$ abnormalities such as partial tandem duplications (MLL-PTD) by direct sequencing [28,29].

\section{Cytogenetics}

For cytogenetic analyses and fluorescence in situ hybridization (FISH) the samples of bone marrow were cultivated for 24 hrs in medium RPMI 1640 with 10\% of fetal calf serum. Twenty G-banded Wright-Giemsa stained mitoses, if available, were evaluated. The karyotypes were described following ISCN 2013 nomenclature. For precise identification of chromosomal aberrations, FISH with locus specific DNA probes (Vysis, Downers Grove, IL, USA) and multicolor FISH with color kit probes and ISIS computer analysis (both from Metasysteme, Altlusheim, Germany) were used.

\section{Data processing and statistics}

Data from targeted bisulfite sequencing were processed and evaluated using freely available programs: (i) FastQC [30] (quality control of reads), (ii) Trimmomatic [31] (removal of adapters/primer-dimers and bases with lowquality scores), (iii) Bismark [32] (methylation-aware alignment of reads to the reference genome and computation of methylation ratios). Differentially methylated target regions 
were assigned to genes using the on-line annotation tool GREAT [11]. Quantile normalization and subtraction of background was applied to the raw microarray expression data in BeadStudio Data Analysis Software (Illumina). Raw data from 454 pyrosequencing were processed using a filter template to relax the stringency of the original valley filter (kindly provided by Dr. Esteban Czwan, Roche). This step was necessary due to the lower complexity of bisulfite treated DNA containing long stretches of homopolymers. The filter template is available on-line (Additional file 10) and its usage is described in Additional file 11. Data from 454 pyrosequencing were aligned to a reference in GS Amplicon Variant Analyzer (AVA) (Roche) software and DNA methylation levels were assessed using the webbased software BISMA [33].

Kaplan-Meier curves and two-sided log-rank test were used to estimate the overall survival and to compare differences between survival curves. The relations between qualitative parameters were compared in contingency tables using Fisher's exact test. For analyses of quantitative data, medians were detected and non-parametric twotailed Mann-Whitney tests were performed. All these tests were conducted at a level of significance of 0.05 using GraphPad Prism4 software (GraphPad Software, San Diego, CA, USA). Cox regression analysis. was performed applying the SPSS statistical software (SPSS Inc., Chicago, IL, USA).

\section{Additional files}

Additional file 1: Figure S1. Inv(16) methylation clusters inside and outside of CGIs. Hierarchal DNA methylation clustering of CpGs inside (A) and outside (B) of CGIs using the correlation as a distance measure and Ward's method (AML_1 to AML_14 - AML patients; CD34_pool - healthy control's CD34+ pool) indicating CBFB-MYH11 methylation cluster (in ellipse) consistently with clustering of all CpGs shown in Figure 1.

Additional file 2: Table S1. Enrichments for inv(16) uniquely differentially methylated regions (DMRs) as observed in GREAT. Enrichment terms observed after uploading of uniquely inv(16) DMRs to on-line annotation tool GREAT [11].

Additional file 3: Table S2. Inv(16) enriched regions. Genomic regions that were enriched for and associated with genes being previously described as upregulated in inv(16) AML.

Additional file 4: Figure S2. Region_2 methylation levels of MN1 and SPARC locus. (A) MN1_region 2 hypomethylation in inv(16) patients compared to AML M4 without inv(16), other AML subtypes and healthy controls; (B) SPARC_region 2 methylation levels are the same when compared AML M4 without inv(16), other AML subtypes and healthy controls.

Additional file 5: Table S3. Genes with strong negative correlation between TSS adjacent DNA methylation levels and their expression. Genes with strong anti-correlation ( $\leq-0.7)$ between DNA methylation and expression, and with change of methylation between control and at least one AML sample 2-fold or greater (with either the control or at least one of the AMLs having methylation ratio 0.3 or greater); 163 genomic regions assigned to 130 unique genes.

Additional file 6: Figure S3. Comparison of $P B X 3$ expression levels. PBX3 relative gene expression levels in AML patients at diagnosis versus healthy controls' samples.
Additional file 7: Table S4. Samples used for inv(16) hypomethylation validation. Characteristics of AML patients examined by 454 bisulfite pyrosequencing to validate inv(16) hypomethylation of selected loci; BM - bone marrow, PB - peripheral blood.

Additional file 8: Table S5. 454 bisulfite primers and annealing temperatures Primer sequences and annealing temperatures used to amplify regions of interest.

Additional file 9: Table S6. TaqMan gene expression assays IDs. IDs of TaqMan probes used to measure gene expression of selected genes.

Additional file 10: Filter template. This template was used for 454 raw sequencing data reprocessing as described in Additional file 10.

Additional file 11: 454 pyrosequencing - raw data acquisition. Description of amplicon filter template usage that allowed to obtain more reads from 454 bisulfite sequencing.

\section{Competing interests}

The authors declare that they have no competing interests.

\section{Authors' contribution}

$\mathrm{HH}$ performed targeted and 454 bisulfite analysis and gene expression assays, analysed and interpreted data and wrote the manuscript; MHYF did the bioinformatics and statistical analyses and wrote the manuscript; $\mathrm{CH}$ interpreted data and wrote the manuscript; JS selected patients for 454 bisulfite sequencing, provided and interpreted clinical data and reviewed the manuscript; CS chose patients for targeted bisulfite analysis, provided and interpreted clinical data and reviewed the manuscript; JM performed molecular genetics analysis and reviewed the manuscript; ZK and MDM performed and evaluated the gene expression microarrays and reviewed the manuscript; AK, MV and OF performed molecular genetics analysis and reviewed the manuscript, KM provided cytogenetic data and reviewed the manuscript; PC provided clinical data and reviewed the article; VB supervised targeted bisulfite analysis and wrote the manuscript. All authors critically reviewed the manuscript and read and approved the final version.

\section{Acknowledgements}

We thank Dinko Pavlinic for help with targeted bisulfite library preparation and Esteban Czwan from Roche for help with raw 454 bisulfite data acquisition. This study was supported by the project (Ministry of Health, (zech Republic) for conceptual development of research organization (00023736, IHBT), by the grant ERDF OPPK CZ.2.16/3.1.00/24001 and was a part of the COST Action BM0801 (EUGESMA).

\section{Author details}

${ }^{1}$ Department of Molecular Genetics, Institute of Hematology and Blood Transfusion, U Nemocnice 1, Prague, Czech Republic. ${ }^{2}$ European Molecular Biology Laboratory (EMBL), Core Facilities and Services, Meyerhofstraße 1, Heidelberg, Germany. ${ }^{3}$ Clinical Department, Institute of Hematology and Blood Transfusion, U Nemocnice 1, Prague, Czech Republic. ${ }^{4}$ Center of Oncocytogenetics, Institute of Medical Biochemistry and Laboratory Diagnostics, General University Hospital and First Faculty of Medicine, Charles University in Prague, U Nemocnice 2, Prague, Czech Republic.

Received: 25 March 2014 Accepted: 6 September 2014 Published online: 30 September 2014

\section{References}

1. Sonnet M, Claus R, Becker N, Zucknick M, Petersen J, Lipka DB, Oakes CC, Andrulis M, Lier A, Milsom MD, Witte T, Gu L, Kim-Wanner S, Schirmacher P, Wulfert M, Gattermann N, Luebbert M, Rosenbauer F, Rehli M, Bullinger L, Weichenhan D, Plass C: Early aberrant DNA methylation events in a mouse model of acute myeloid leukemia. Genome Med 2014, 6:34.

2. Figueroa ME, Lugthart S, Li Y, Erpelinck-Verschueren C, Deng X, Christos PJ, Schifano E, Booth J, van Putten W, Skrabanek L, Campagne F, Mazumdar M, Greally JM, Valk PJM, Lowenberg B, Delwel R, Melnick A: DNA methylation signatures identify biologically distinct subtypes in acute myeloid leukemia. Cancer Cell 2010, 17(1):13-27.

3. Cancer Genome Atlas Research Network: Genomic and epigenomic landscapes of adult de novo acute myeloid leukemia. N Engl J Med 2013, 368(22):2059-2074. 
4. Akalin A, Garrett-Bakelman FE, Kormaksson M, Busuttil J, Zhang L, Khrebtukova I, Milne TA, Huang Y, Biswas D, Hess JL, Allis CD, Roeder RG, Valk PJM, Lowenberg B, Delwel R, Fernandez HF, Paietta E, Tallman MS, Schroth GP, Mason CE, Melnick A, Figueroa ME: Base-pair resolution DNA methylation sequencing reveals profoundly divergent epigenetic landscapes in acute myeloid leukemia. PLoS Genet 2012, 8(6):e1002781.

5. Jones PA: Functions of DNA methylation: islands, start sites, gene bodies and beyond. Nat Rev Genet 2012, 13(7):484-492.

6. Ivanov M, Kals M, Kacevska M, Metspalu A, Ingelman-Sundberg M, Milani L: In-solution hybrid capture of bisulfite-converted DNA for targeted bisulfite sequencing of 174 ADME genes. Nucleic Acids Res 2013, 41(6):e72.

7. Sproul D, Kitchen RR, Nestor CE, Dixon JM, Sims AH, Harrison DJ, Ramsahoye $\mathrm{BH}$, Meehan RR: Tissue of origin determines cancer-associated CpG island promoter hypermethylation patterns. Genome Biol 2012, 13(10):R84.

8. Valk P, Verhaak R, Beijen M, Erpelinck C, van Doorn-Khosrovani S, Boer J, Beverloo H, Moorhouse M, van der Spek $\mathrm{P}$, Lowenberg B, Delwel R: Prognostically useful gene-expression profiles in acute myeloid leukemia. N Engl J Med 2004, 350(16):1617-1628

9. Li Z, Zhang Z, Li Y, Arnovitz S, Chen P, Huang H, Jiang X, Hong G, Kunjamma RB, Ren H, He C, Wang C, Elkahloun AG, Valk PJM, Doehner K Neilly MB, Bullinger L, Delwel R, Lowenberg B, Liu PP, Morgan R, Rowley JD, Yuan C, Chen J: PBX3 is an important cofactor of HOXA9 in leukemogenesis. Blood 2013, 121(8):1422-1431.

10. Suzuki R, Shimodaira H: Pvclust: an R package for assessing the uncertainty in hierarchical clustering. Bioinformatics 2006, 22(12):1540-1542.

11. McLean CY, Bristor D, Hiller M, Clarke SL, Schaar BT, Lowe CB, Wenger AM, Bejerano G: GREAT improves functional interpretation of cis-regulatory regions. Nat Biotechnol 2010, 28(5):495-U155.

12. Dickson GJ, Liberante FG, Kettyle LM, O'Hagan KA, Finnegan DPJ, Bullinger L, Geerts D, McMullin MF, Lappin TRJ, Mills KI, Thompson A: HOXA/PBX3 knockdown impairs growth and sensitizes cytogenetically normal acute myeloid leukemia cells to chemotherapy. Haematologica 2013, 98(8):1216-1225.

13. de la Bletiere DR, Blanchet $O$, Cornillet-Lefebvre $P$, Coutolleau A, Baranger $L$, Genevieve F, Luquet I, Hunault-Berger M, Beucher A, Schmidt-Tanguy A, Zandecki M, Delneste Y, Ifrah N, Guardiola P: Routine use of microarray-based gene expression profiling to identify patients with low cytogenetic risk acute myeloid leukemia: accurate results can be obtained even with suboptimal samples. BMC Med Genet 2012, 5:6.

14. Mandoli A, Singh AA, Jansen PWTC, Wierenga ATJ, Riahi H, Franci G, Prange K, Saeed S, Vellenga E, Vermeulen M, Stunnenberg HG, Martens JHA: CBFB-MYH11/ RUNX1 together with a compendium of hematopoietic regulators, chromatin modifiers and basal transcription factors occupies self-renewal genes in inv (16) acute myeloid leukemia. Leukemia 2014, 28(4):770-778.

15. Heuser M, Beutel G, Krauter J, Doehner K, von Neuhoff N, Schlegelberger B, Ganser A: High meningioma 1 (MN1) expression as a predictor for poor outcome in acute myeloid leukemia with normal cytogenetics. Blood 2006, 108(12):3898-3905.

16. Langer C, Marcucci G, Holland KB, Radmacher MD, Maharry K, Paschka P, Whitman SP, Mrozek K, Baldus CD, Vij R, Powell BL, Carroll AJ, Kolitz JE, Caligiuri MA, Larson RA, Bloomfield CD: Prognostic importance of MN1 transcript levels, and biologic insights from MN1-associated gene and MicroRNA expression signatures in cytogenetically normal acute myeloid leukemia: a cancer and leukemia group B study. J Clin Oncol 2009, 27(19):3198-3204

17. Grimwade D, Walker H, Oliver F, Wheatley K, Harrison C, Harrison G, Rees J, Hann I, Stevens R, Burnett A, Goldstone A, Med Res Council Adult Children's Leukaemia Work: The importance of diagnostic cytogenetics on outcome in AML: analysis of 1,612 patients entered into the MRC AML 10 trial. Blood 1998, 92(7):2322-2333.

18. Mrózek K, Bloomfield CD: Clinical significance of the most common chromosome translocations in adult acute myeloid leukemia. JNCI Monogr 2008, 2008(39):52-57.

19. Carella C, Bonten J, Sirma S, Kranenburg TA, Terranova S, Klein-Geltink R, Shurtleff S, Downing JR, Zwarthoff EC, Liu PP, Grosveld GC: MN1 overexpression is an important step in the development of inv(16) AML. Leukemia 2007, 21(8):1679-1690.

20. Chang C, Brocchieri L, Shen W, Largman C, Cleary M: Pbx modulation of Hox homeodomain amino-terminal arms establishes different DNA-binding specificities across the Hox locus. Mol Cell Biol 1996, 16(4):1734-1745.
21. Itonaga $H$, Imanishi $D$, Wong $Y$, Sato $S$, Ando $K$, Sawayama $Y$, Sasaki D, Tsuruda K, Hasegawa H, Imaizumi Y, Taguchi J, Tsushima H, Yoshida S, Fukushima T, Hata T, Moriuchi Y, Yanagihara K, Miyazaki Y: Expression of myeloperoxidase in acute myeloid leukemia blasts mirrors the distinct DNA methylation pattern involving the downregulation of DNA methyltransferase DNMT3B. Leukemia 2014, 28(7):1459-1466.

22. Schneider M, Szaumkessel M, Richter J, Ammerpohl O, Hansmann ML, Küppers R, Siebert R, Giefing M: The PRDX2 gene is transcriptionally silenced and de novo methylated in Hodgkin and Reed-Sternberg cells of classical Hodgkin lymphoma. Blood 2014, 123(23):3672-3674.

23. Jiang D, Hong Q, Shen Y, Xu Y, Zhu H, Li Y, Xu C, Ouyang G, Duan S: The diagnostic value of DNA methylation in leukemia: a systematic review and meta-analysis. PLoS One 2014, 9(5):e96822.

24. Markova J, Trnkova Z, Michkova P, Maaloufova J, Stary J, Cetkovsky P, Schwarz J: Monitoring of minimal residual disease in patients with core binding factor acute myeloid leukemia and the impact of C-KIT, FLT3, and JAK2 mutations on clinical outcome. Leuk Lymphoma 2009, 50(9):1448-1460.

25. Pitiot AS, Santamaria I, Garcia-Suarez O, Centeno I, Astudillo A, Rayon C, Balbin M: A new type of NPM1 gene mutation in AML leading to a C-terminal truncated protein. Leukemia 2007, 21(7):1564-1566.

26. Fuchs O, Provamikova D, Kocova M, Kostecka A, Cvekova P, Neuwirtova R, Kobylka P, Cermak J, Brozinova J, Schwarz J, Markova J, Salaj P, Klamova H, Maaloufova J, Lemez P, Novakova L, Benesova K: CEBPA polymorphisms and mutations in patients with acute myeloid leukemia, myelodysplastic syndrome, multiple myeloma and non-Hodgkin's lymphoma. Blood Cells Mol Dis 2008, 40(3):401-405.

27. Markova J, Michkova P, Burckova K, Brezinova J, Michalova K, Dohnalova A, Maaloufova JS, Soukup P, Vitek A, Cetkovsky P, Schwarz J: Prognostic impact of DNMT3A mutations in patients with intermediate cytogenetic risk profile acute myeloid leukemia. Eur J Haematol 2012, 88(2):128-135.

28. Caligiuri M, Strout M, Schichman S, Mrozek K, Arthur D, Herzig G, Baer M, Schiffer C, Heinonen K, Knuutila S, Nousiainen T, Ruutu T, Block A, Schulman $P$, PedersenBjergaard J, Croce C, Bloomfield C: Partial tandem duplication of ALL1 as a recurrent molecular defect in acute myeloid leukemia with trisomy 11. Cancer Res 1996, 56(6):1418-1425.

29. Shiah $H$, Kuo $Y$, Tang J, Huang S, Yao M, Tsay W, Chen Y, Wang C, Shen M, Lin D, Lin K, Tien H: Clinical and biological implications of partial tandem duplication of the MLL gene in acute myeloid leukemia without chromosomal abnormalities at 11q23. Leukemia 2002, 16(2):196-202.

30. Krueger F, Kreck B, Franke A, Andrews SR: DNA methylome analysis using short bisulfite sequencing data. Nat Methods 2012, 9(2):145-151.

31. Lohse $M$, Bolger AM, Nagel A, Fernie AR, Lunn JE, Stitt M, Usadel B: RobiNA: a user-friendly, integrated software solution for RNA-Seq-based transcriptomics. Nucleic Acids Res 2012, 40(W1):W622-W627.

32. Krueger F, Andrews SR: Bismark: a flexible aligner and methylation caller for Bisulfite-Seq applications. Bioinformatics 2011, 27(11):1571-1572.

33. Rohde C, Zhang Y, Reinhardt R, Jeltsch A: BISMA - Fast and accurate bisulfite sequencing data analysis of individual clones from unique and repetitive sequences. BMC Bioinformatics 2010, 11:230.

\section{doi:10.1186/s13045-014-0066-4}

Cite this article as: Hájková et al:: CBFB-MYH11 hypomethylation signature and $P B X 3$ differential methylation revealed by targeted bisulfite sequencing in patients with acute myeloid leukemia. Journal of Hematology \& Oncology 2014 7:66. 